\title{
Analyse des pratiques d'hygiène et de fabrication et évaluation de la qualité du Mashanza dans 12 unités de production au Sud-Kivu
}

\author{
Birali Mwamini, ${ }^{1,2}$, Sumbu Zola ${ }^{2}$, Walangululu Jean ${ }^{1}$, Busime Munamire ${ }^{1}$ et Cirhuza \\ Matabaro ${ }^{1}$ \\ ${ }^{1}$ Université Catholique de Bukavu (UCB), Faculté des Sciences agronomiques, BP. 02, Cyangugu, Rwanda, \\ ${ }^{2}$ Université de Kinshasa (UNIKIN), Faculté d'Agronomie, B.P. 117, Kinshasa I, RD. Congo. \\ Corresponding Author : Birali, M.; 1; +243994646409
}

Mots-clés : Mashanza, pratique, fabrication, hygiène, qualité

Keywords: Mashanza, practice, manufacturing, hygiene, quality

Publication date 31/12/2019, http://www.m.elewa.org/JAPS

\section{RESUME}

Le Mashanza est un fromage frais traditionnel produit à l'Est de la RDC particulièrement au Sud-Kivu. Malheureusement, sa production est demeurée artisanale, malgré son importance commerciale et les pratiques différant d'un endroit à un autre. L'objet de la présente étude est de diagnostiquer l'état sanitaire et les pratiques de fabrication de ce produit. 12 unités de fabrication localisées dans les territoires de Kabare, Kalehe, Uvira et Walungu ont servi de sujet d'études. La méthode des $5 \mathrm{M}$ a permis de classer les divers facteurs susceptibles de contribuer à la dépréciation de la qualité du Mashanza. Les résultats ont montré que la majorité des unités de fabrication sont de type artisanal et ne sont pas conformes sur le plan des installations et de l'assainissement. Les équipements utilisés sont rudimentaires. Le personnel est peu instruit et n'a pas été formé aux bonnes pratiques de fabrication et d'hygiène. Les résultats de l'analyse physico-chimique des produits montrent des valeurs comprises entre $31,1 \%$ et $48,8 \%$ pour la matière sèche, 3,5 et 3,9 pour le $\mathrm{pH}$, de 0,87 et 0,95 pour l'Aw et de 41,5 et $100,8^{\circ} \mathrm{D}$ pour l'acidité, traduisant entre autres l'influence de la variabilité des procédés sur la qualité du Mashanza. L'analyse microbiologique révèle une flore aérobie mésophile totale variant entre 4 et $8.102 \mathrm{ufc} / \mathrm{ml}$ pour le lactosérum et 0 et 9,8.10 $\mathrm{ufc} / \mathrm{g}$ pour le Mashanza. Le lactosérum accuse la présence des coliformes : E.coli, S.aureus, les germes anaérobies sulfito-réducteurs, les levures et les moisissures. Leur dénombrement montre des valeurs variant entre 0 et $1,4.10^{2} \mathrm{ufc} / \mathrm{g}$ pour les coliformes totaux, 0 et $36 \mathrm{ufc} / \mathrm{g}$ pour les coliformes fécaux, 0 et 1,6.10 $\mathrm{ufc} / \mathrm{g}$ pour $\mathrm{E}$. coli; 0 et 1,2.103, ufc $/ \mathrm{ml}$ pour les anaérobies sulfito-réducteurs et entre 0 et 1,5.103. ufc/g pour les levures et les moisissures. Salmonella sp. est absent de tous les échantillons de lactosérum et de Mashanza. En général, de « Mashanza » est satisfaisante. Les résultats obtenus permettent d'orienter les producteurs et les chercheurs sur les facteurs pouvant déprécier la qualité du Mashanza et sur lesquels on peut agir. 


\begin{abstract}
Mashanza is a traditional fresh cheese produced in eastern DRC, particularly in South Kivu. Unfortunately, its production has remained artisanal, despite its commercial importance and practices differ from one place to another. The purpose of this study is to diagnose the health status and manufacturing practices of this product. 12 manufacturing units located in the Kabare, Kalehe, Uvira and Walungu territories were used as subjects of study. The 5M method classified the various factors that may contribute to the depreciation of the quality of the Mashanza. The results showed that the majority of manufacturing units are of the artisanal type and are not compliant in terms of facilities and sanitation. The equipment used is rudimentary. The staff is poorly educated and has not been trained in good manufacturing and hygiene practices. The results of the physicochemical analysis of the products show values between $31.1 \%$ and $48.8 \%$ for the dry matter, 3.5 and 3.9 for the $\mathrm{pH}$, of 0.87 and 0.95 for $\mathrm{Aw}$ and 41.5 and $100.8^{\circ} \mathrm{D}$ for acidity, reflecting, among other things, the influence of process variability on Mashanza quality. The microbiological analysis reveals a total mesophilic aerobic flora varying between 4 and $8 \times 102 \mathrm{cfu} / \mathrm{ml}$ for whey and 0 and 9.8 $\times 102 \mathrm{cfu} / \mathrm{g}$ for Mashanza. The whey accuses the presence of coliforms: $E$. coli, S.aureus, anaerobic sulphite-reducing germs, yeasts and moulds. Their enumeration shows values ranging between 0 and $1.4 \times 102 \mathrm{cfu} / \mathrm{g}$ for total coliforms, 0 and $36 \mathrm{cfu} / \mathrm{g}$ for faecal coliforms, 0 and $1.6 \times 102 \mathrm{cfu} / \mathrm{g}$ for E. coli; 0 and 1.2.103, cfu / $\mathrm{ml}$ for the sulphitoreducing anaerobes and between 0 and 1.5.103. ufc / $\mathrm{g}$ for yeasts and moulds. Salmonella sp. is absent from all whey and Mashanza samples. In general, Mashanza is satisfactory. The results obtained make it possible to guide producers and researchers on the factors that can degrade the quality of Mashanza and that can be acted on.
\end{abstract}

\section{INTRODUCTION}

A l'est de la RDC, la technologie traditionnelle de production du fromage frais, communément appelé Mashanza, occupe une place importante dans la transformation artisanale du lait frais. La fabrication du Mashanza est issue d'un égouttage lent étant donné son mode de production jusqu'alors traditionnel ne recourant pas aux équipements sophistiqués avec comme inconvénient une rétention élevée d'humidité si le processus d'égouttage n'est pas prolongé ou mené correctement. En effet, le contenu élevé en eau libre contribue essentiellement aux difficultés de conservation du «Mashanza», contrairement aux autres types de fromages dont la teneur en matière sèche est élevée suite à un égouttage poussé, ou à un pressage pour l'obtention d'un produit compacté. Ramet (1985) montre que la stabilisation des fromages résulte essentiellement l'de l'abaissement de $\mathrm{pH}$ et de l'activité de l'eau. Les fromages blancs obtenus par la voie lactique sont caractérisés par un coagulum dont le $\mathrm{pH}$ est inférieur à 4,5 et une teneur en matière sèche comprise entre 20 et $25 \%$ (FAO, 1998). L'activité de l'eau se trouve dans l'intervalle 0,980 à 0,995 (Mahaut et al., 2000). Des durées de vie de moins d'une semaine ont été rapportées par plusieurs auteurs (FAO, 1990; Cirhuza \& Bufole cités par Birali ${ }^{1}$ et al., 2019 ; Birali ${ }^{2}$ et al., 2019). Dans certaines zones de production, au Sud-Kivu, particulièrement, l'absence ou l'irrégularité de la fourniture en énergie électrique est à la base des difficultés de conservation de la plupart des denrées alimentaires périssables comme c'est le cas pour le lait et les produits laitiers. De plus, à part le manque d'uniformité des procédés d'un endroit à un autre pouvant conduire à des produits de qualité diversifiée, la production du Mashanza est demeurée artisanale. Les équipements utilisés et les pratiques d'usage en cours de fabrication, du conditionnement et du nettoyage des équipements peuvent, également, occasionner une perte de qualité et limiter la promotion de ce produit et sa consommation à 
l'échelle locale et par les traditionnalistes compte tenu des exigences de plus en plus strictes des consommateurs et du marché. La normalisation contribue à une nécessité sanitaire et économique par l'établissement des règles visant à garantir qualité et sécurité aux consommateurs (Sylvander, 1996). Elle a comme avantage l'homogénéisation de la qualité des produits et la responsabilité légale des acteurs (Poret, 2011). Les normes portent sur des domaines vastes et variés tels: la terminologie, les compositions qualitative et quantitative, les matériaux de contact avec les aliments, etc. (Roudaut et Lefrancq, 2005).
L'objectif de cette étude est d'analyser les pratiques d'hygiène, de fabrication et la qualité du Mashanza produit par 12 unités de fabrication localisées à Kabare, Kalehe, Uvira et Walungu en vue de sa normalisation et sa stabilisation. Elle vise spécifiquement à :

- décrire les conditions de production du Mashanza ;

- identifier les facteurs pouvant affecter les paramètres qualitatifs du Mashanza ;

- évaluer la qualité du produit sur le plan physico-chimique et microbiologique.

\section{MILIEU, MATÉRIELS ET MÉTHODES}

3.1 Milieu d'étude : l'étude a été réalisée de septembre à novembre 2018 dans la province du Sud-Kivu à l'est de la RD Congo, située entre $1^{\circ} 36^{\prime}$ et $5^{\circ}$ de latitude sud et $26^{\circ} 47^{\prime}$ et $29^{\circ} 20^{\prime}$ de longitude est (ministère du plan, 2005). L'échantillonnage a été réalisé dans 4 territoires (Kabare, Kalehe, Uvira et Walungu). 12 unités de production ont été ciblées à la suite d'une étude antérieure. Le tableau 1 donne les coordonnées géographiques des unités de fabrication.

Tableau 1 : Coordonnées géographiques des 12 unités de fabrication.

\begin{tabular}{|c|c|c|}
\hline Nom & Territoire & Coordonnées géographiques \\
\hline $\mathrm{Ucb}$ & Kabare & Altitude : $1701 \mathrm{~m} ;$ Latitude : $02.28977^{\circ}$; Longitude: $028.82176^{\circ}$ \\
\hline Comis & Kabare & Altitude : $1706 \mathrm{~m}$; Latitude:02.28716º ; Longitude:028.82007 \\
\hline Miti & Kabare & Altitude : $1584 \mathrm{~m}$; Latitude: $02.36128^{\circ}$; Longitude: $028.79142^{\circ}$ \\
\hline Kond & Uvira & Altitude : 945m ; Latitude:02.90645 ; Longitude:029.05540 \\
\hline Zia & Uvira & Altitude : $972 \mathrm{~m}$; Latitude:02.542193 ; Longitude:029.031515 \\
\hline Shuk & Uvira & Altitude : $945 \mathrm{~m}$; Latitude:02.542316 ; Longitude:029.031385 \\
\hline Luk & Kalehe & Altitude : $1480 \mathrm{~m} ;$ Latitude : $02.653^{\circ}$; Longitude : $028.91905^{\circ}$ \\
\hline Mag & Kalehe & Altitude : $1478 \mathrm{~m} ;$ Latitude : 02.11727 ; Longitude : $028.89541^{\circ}$ \\
\hline Luz & Kalehe & Altitude: $1507 \mathrm{~m}$; Latitude:02.122265 ; Longitude:029.87343 \\
\hline Cing & Walungu & Altitude : $2400 \mathrm{~m}$; Latitude:02.49273; Longitude:028.69902º \\
\hline $\mathrm{Cab}$ & Walungu & Altitude : $2474 \mathrm{~m}$; Latitude: $02.51944^{\circ}$; Longitude:028.68505 \\
\hline Iprelo & Walungu & 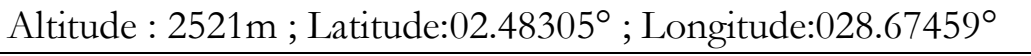 \\
\hline
\end{tabular}

3.2 Matériels : Pour l'enquête, le questionnaire a été utilisé. Par ailleurs, les échantillons de Mashanza et de lactosérum ont été prélevés dans chaque ferme, puis analysés.

3.3 Méthodes : Dans cette étude, deux approches ont été suivies : celle basée sur des enquêtes formelles faisant usage du questionnaire et celle basée sur l'expérimentation en faisant des analyses physico-chimiques et microbiologique du Mashanza et du lactosérum. L'échantillonnage a été réalisé à Kabare, Kalehe, Uvira et Walungu où la fabrication du Mashanza est ancrée dans la tradition des communautés. Les principaux critères de choix ont été les suivants: (i) ancienneté dans la production du 
«Mashanza», (ii) pérennité et viabilité de l'activité toute l'année, (iii) capacité de traitement d'au moins 20 litres par opération ou par jour, (iv) accessibilité de la ferme par véhicule, (v) proximité de la route principale, (vi) distance de moins de 30 minutes en véhicule, d'une ferme à une autre dans le même territoire. Les défauts potentiellement présents dans les denrées alimentaires, peuvent provenir de 5 sources possibles de contamination que l'on peut étudier à partir des « 5M » (Quittet \& Nelis, 1999). Ainsi, l'analyse des écarts liés à l'hygiène a été réalisée grâce au diagramme de cause-effet ou en arête de poisson en classant les pratiques et les causes en 5 grandes familles (5M) dont le Milieu, Matériel, Main-d'œuvre, Matière et Méthode (procédé). Quant aux analyses physico-chimiques et microbiologiques du Mashanza et du lactosérum, elles se sont déroulées respectivement au laboratoire de Science du sol de l'Université Catholique de Bukavu et au laboratoire de Microbiologie de l'Office Congolais de Contrôle (OCC/Bukavu). Après prélèvement, les échantillons de Mashanza ont été conservés dans les sachets stériles, puis réfrigérés à $4^{\circ} \mathrm{C}$ en attendant les analyses et ceux de lactosérum, dans des bouteilles en plastique dans les mêmes conditions.

2.3.1 Analyse des pratiques d'hygiène et de fabrication: Un questionnaire d'enquête a été soumis aux fabricants du Mashanza dans 12 unités de transformation réparties dans les 4 territoires en étude en vue de collecter les informations générales et spécifiques en rapport avec l'unité de fabrication et les procédures lors de la fabrication de Mashanza, incluant la traite ainsi que les équipements utilisés.

2.3.2 Analyse des paramètres physicochimiques: Le $\mathrm{pH}$ a été déterminé par la méthode potentiométrique et consistante à plonger l'électrode dans la solution de l'échantillon de lactosérum ou dans la solution homogénéisée de $5 \mathrm{~g}$ de Mashanza avec de l'eau distillée et à lire la valeur de $\mathrm{pH}$ sur l'écran du pH-mètre. Le taux de matières sèches a été obtenu après pesée des échantillons frais et ceux séchées à l'étuve à $103^{\circ} \mathrm{C} 24$ heures et correspondant au rapport de l'échantillon frais et sec exprimé en pourcents. L'activité de l'eau (Aw) a été déterminée à l'aide de l'appareil LabStart-aw de marque Novasina. Pour chaque mesure, l'échantillon est placé dans la coupelle et déposé dans la chambre de mesure en prenant soin de refermer le capot. Ensuite le bouton de démarrage est enclenché et la valeur est affichée sur l'écran. L'acidité Dornic a été obtenu par titration de $10 \mathrm{~g}$ d'échantillon mélangé à $100 \mathrm{ml}$ d'eau distillée avec du $\mathrm{NaOH}$ en utilisant le phénolphtaléine $(1 \mathrm{ml}$ comme indicateur coloré).

2.3.3 Analyse des paramètres microbiologiques : Les paramètres microbiologiques sont été évalués en se référant au manuel suisse des denrées alimentaires suivi par le laboratoire de l'O.C.C. Les milieux de culture et les températures et durées d'incubation pour chaque type de microorganismes sont définis dans le tableau 2.

Tableau 2:Milieu de culture utilisé dans la recherche des microorganismes Milieu de culture et température d'incubation Organisme recherché 


\begin{tabular}{|l|l|}
\hline Plate Count Agar (PCA) à $37^{\circ} \mathrm{C}$ & Flore aérobie mésophile totale \\
\hline Violet Red Bile Glucose Agar (VRB) à $37^{\circ} \mathrm{C}$ & Coliformes totaux \\
\hline Violet Red Bile Glucose Agar (VRB) à $44^{\circ} \mathrm{C}$ & Coliformes fécaux \\
\hline Eosine Bleue de Méthyle (EMB) à $37^{\circ} \mathrm{C}$ & Escherichia coli \\
\hline Manitol Sat Agar (MSA) à $37^{\circ} \mathrm{C}$ & Staphylococcus aureus \\
\hline SPS à $37^{\circ} \mathrm{C}$ & Anaérobies sulfito- réducteurs \\
\hline BHI à $22^{\circ} \mathrm{C}$ & Levures et moisissures \\
\hline LACTO à $37^{\circ} \mathrm{C}$ & Lactobacillus spp \\
\hline Saburraux au Chloramphénicol (XLD) à $37^{\circ} \mathrm{C}$ & Salmonella spp \\
\hline
\end{tabular}

3.3.4 Analyse statistique : Les analyses des données d'enquêtes et celles des paramètres physico-chimiques ont été réalisées par la

\section{RÉSULTATS ET DISCUSSION}

4.1 Caractéristiques des unités de transformation: La figure 1 donne le Pourcentage des unités de fabrication dans les 4 territoires en fonction du \% des enquêtés, du type d'unité et des personnes travaillant dans la fromagerie. Il ressort des résultats que toutes les unités des transformations étudiées sont artisanales ou traditionnelles. La main d'œuvre utilisée est variable: 33,3\% d'unités emploient respectivement 1 et 2 personnes tandis que $16,7 \%$ ont respectivement 3 et 4 personnes. Aucune unité enquêtée n'employant plus de 4 personnes. Ceci se justifie dans la mesure où les unités possèdent une faible capacité de production et étant donné leur caractère artisanal dans la majorité des cas ne nécessitant pas un effectif important.

4.2 Etat hygiénique tout au long de la chaîne de fabrication du Mashanza et analyse cause-effet suivant la méthode d'ishikawa ou méthode 5M: La figure 4 statistique descriptive grâce aux logiciels IBM Statistics SPSS 16 et Excel.

résume les problèmes rencontrés dans les unités de production étudiées et classés selon le diagramme en arête de poisson ou méthode 5 M.

4.2.1 Milieu : Les résultats de la figure 1 ont révélé que $50 \%$ des bâtiments de fabrication du Mashanza ont des sols en terre. A Kabare, par contre, tous les ateliers disposent des revêtements recouvrant le sol. Par ailleurs, $100 \%$ de bâtiments ont des portes et des fenêtres en planches et la majorité des bâtiments ont des plafonds en tôle, soit 75\% et seulement $25 \%$ des bâtiments possèdent des plafonds en paille. Concernant l'usage des bâtiments, $66,7 \%$ de ces derniers sont soumis à la fumée et $33,3 \%$ ne le sont pas. En outre, $50 \%$ des bâtiments de fabrication servent d'habitation humaine. De même, 100\% des bâtiments n'ont pas de réseaux d'évacuation d'eaux usées. 


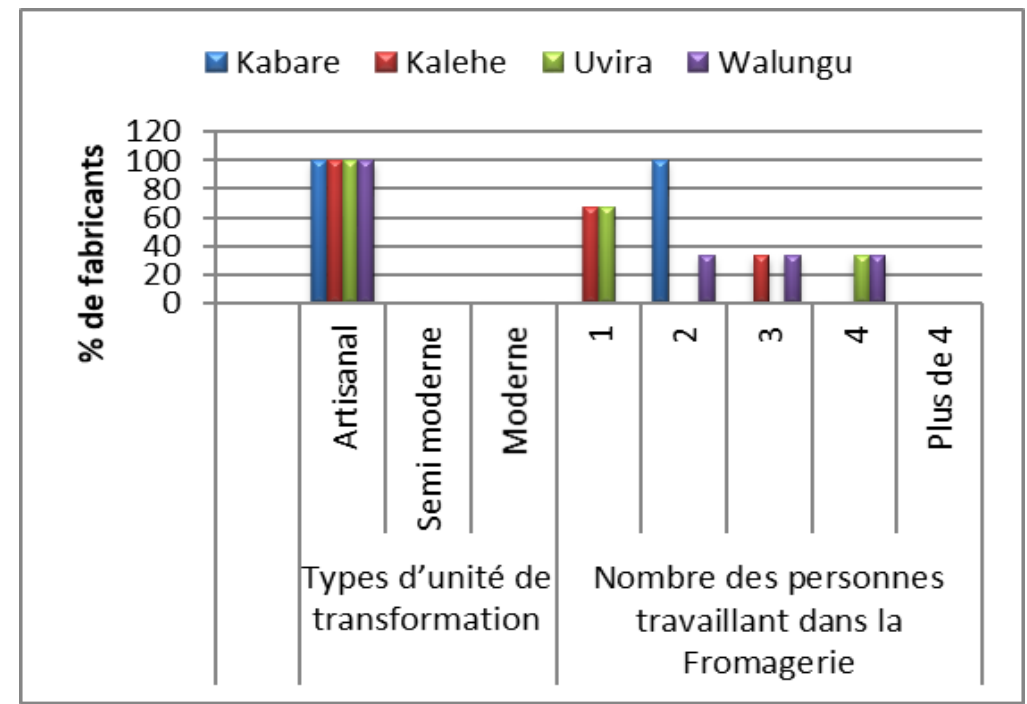

Figure 1: Pourcentage des unités de fabrication dans les 4 territoires en fonction du \% des enquêtés, du type d'unité et des personnes travaillant dans la fromagerie

Selon l'Arrêté royal du 7 mars 1994 relatif à l'agrément des établissements laitiers, les sols des bâtiments de transformation des produits laitiers doivent être faits de matériaux, résistants, durables, étanches et nettoyables ne pouvant pas entrainer la contamination de l'environnement ou des aliments (Quittet \& Nelys, 1999). Quant aux structures se trouvant à l'intérieur des établissements de production alimentaire, elles devraient être construites solidement en matériaux durables et être faciles à entretenir, à nettoyer et à désinfecter (FAO, 2011). Dans les locaux d'usage de fabrication des produits sensibles aux développements microbiens, étant donné le rôle de l'air comme vecteur de contamination particulaire et microbienne, la température est maintenue entre 6 et $12^{\circ} \mathrm{C}$ et l'hygrométrie à un niveau compatible avec l'activité (Bimbenet et al., 2007). Par ailleurs, les zones d'odeur désagréable, de fumée, de poussières et de contaminants sont proscrites pour les établissements laitiers (Quittet et Nelis, 1999). Dans cette étude, la présence de la fumée peut résulter de l'usage de bois comme combustible pour la cuisson. Enfin, Quittet \& Nelis (1999) suggèrent d'éviter la stagnation de l'eau, sauf lorsqu'elle est volontairement recherchée par le mouillage du sol, pour des raisons technologiques.

4.2.2 Matériel : Les résultats ont révélé que les fabricants enquêtés traient le lait dans un récipient en plastique (bidon coupé, seau en plastique, etc.) soit $83,6 \%$ et seulement $16,7 \%$ traient dans un récipient en métal (seau, cruche), sans doute parce que les équipements en plastiques sont plus accessibles du point de vue disponibilité et coût. Par ailleurs, la filtration du lait avant inoculation est réalisée seulement par 58,3\%. Ceux qui ne procèdent pas à la filtration n'ont pas donné de raison à cela. Le matériel de manutention du lait est l'une des plus importantes sources de contamination microbienne dans le lait (Ndungu et al, 2016). Les jerricans en plastique peuvent contribuer à la détérioration de la qualité du lait comme l'ont démontré Orregård et Gemechu et al. cités par Ndungu et al. (2016) qui ont découvert que les producteurs de lait utilisent des récipients en plastique difficiles à nettoyer et à désinfecter occasionnant une manipulation moins hygiénique comparée à l'utilisation de contenants en aluminium. Le matériel de cuisson est principalement la casserole. $41,7 \%$ de fabricants chauffent le lait, sans couvercle. Cependant, le manque de 
contrôle du barème de pasteurisation ou de thermisation est une faille dans l'optique de l'optimisation de la qualité. Selon Anonyme (2009), la cuisson du lait doit se faire dans un récipient couvert afin de protéger le contenu contre l'introduction de microorganismes pendant et après le traitement par la chaleur. $100 \%$ de fabricants, dans tous les territoires, inoculent le lait dans la cuve de fermentation. Les cuves sont, soit en métal et sont hermétiquement clos pour $8,3 \%$ de fabricants à Walungu, soit en plastique pour $100 \%$ de fabricants respectivement à Kabare et Kalehe, $66,7 \%$ à Uvira et 33,3\% à Walungu utilisant des seaux couverts et 33,3\% à Uvira utilisant des bassins non couverts; soit en bois en utilisant des calebasses non hermétiquement fermés correspondant à $33,3 \%$ de fabricants à Walungu. Konté (1999) recommande le remplacement des ustensiles traditionnels, chaque fois que les bénéfices le permettront, par ceux en fer émaillé, en aluminium et même en plastique. En outre, la majorité des enquêtés font l'égouttage lent, à l'aide d'un tank muni de vanne, soit $58,3 \% ; 25 \%$ le font à la main et $16,7 \%$ le font à l'aide d'un bâton en bois. Concernant le mode d'égouttage, il dépend de l'équipement utilisé, du milieu et surtout des quantités de lait disponibles. Par conséquent, ces différents modes d'égouttage peuvent influencer la variabilité de la qualité compte tenu de la teneur en eau et des éléments nutritifs résiduels dans le Mashanza résultant des différences dues aux matériels utilisés et à la pression développée, mais aussi à l'état hygiénique des surfaces de contact avec le produit. Concernant la conservation, la majorité des fabricants conservent le Mashanza dans un récipient hermétiquement clos. Par contre, pour la conservation du ferment, $58,2 \%$ de producteurs emploient des récipients non couverts (bidon, bassin) tandis que 41,7\% utilisent des récipients couverts hermétiquement. Concernant le nettoyage des équipements, seulement 8,3\% recourent à l'eau chaude et au savon pour nettoyer le matériel tandis que le reste utilise de l'eau froide et du savon. Pour enlever la salissure, le frotteur, généralement en sac, est employé seul ou accompagné d'herbes sauvages ou de sable fin pour le récurage. Les opérations de nettoyage, de désinfection et de rinçage ont pour but, respectivement d'éliminer les souillures, de détruire les microorganismes et d'éliminer les résidus des produits chimiques utilisés. Les méthodes et le matériel de nettoyage dépendront de la nature de l'entreprise alimentaire (FAO, 2011). Selon Bimbenet \& Cie (2007), les opérations de nettoyage-désinfection mettent en jeu 3 types d'action: mécaniques, thermiques et chimiques. Ainsi d'une manière générale, le matériel et les conteneurs réutilisables doivent garantir un nettoyage, une désinfection et un entretien convenables pour éviter la contamination et doivent être exempts d'effet toxique pour l'usage auquel ils sont destinés (FAO, 2011). Toutefois, l'efficacité des substances naturelles employées par les producteurs interrogés, telles les herbes utilisées, devraient être étudiée afin d'évaluer également si elles ne représentent aucun danger pour le produit et le consommateur.

4.2.3 Méthodes (Procédures): A Kabare, Kalehe et Walungu où les ateliers de fabrication sont logés au sein des fermes, pour $88,9 \%$ des enquêtés, la traite est effectuée deux fois par jour et seuls $11,1 \%$ traient une fois par jour. Le lait est continuellement ajouté dans la cuve jusqu'à la fin de la fermentation. Le délai d'intervalle entre les deux lactations doit être de 12 heures pour avoir la même quantité le matin et le soir (Anonyme, 1993). Ainsi, le mélange de lait a pour but de conserver la qualité du lait et de ralentir la prolifération des espèces bactériennes présentes, essentiellement la flore mésophile lactique, responsable de l'acidification du lait, et la flore pathogène ou nuisible (Anonyme 2009). Il ressort aussi que la majorité des enquêtés $(55,6 \%)$ ne nettoient pas les mamelles des vaches avant la traite et seulement $41,7 \%$ d'enquêtés filtrent le lait avant inoculation afin d'éliminer les impuretés dans le lait. Concernant la pasteurisation, 41,7\% des enquêtés pasteurisent le lait contre 58,3\% qui ne pasteurisent pas. Selon eux, le chauffage s'effectue à des durées variables, allant de 
quelques minutes à 20 minutes, jusqu'à la formation d'une mousse ou d'une écume à la surface du lait. En France, le décret N 88-1206 du 30 décembre 1988 exige que les matières premières employées pour la fabrication des fromages blancs et des fromages frais, sauf exception prévue, doivent avoir subi un traitement thermique équivalent à la pasteurisation (Goudédranche et al., 2001). Des traitements thermiques modérés $\left(72^{\circ} \mathrm{C}\right.$ pendant 15 à 20 secondes; $63^{\circ} \mathrm{C}$ pendant 15 à 20 secondes) sont recommandés pour réduire les risques relatives à la perte d'aptitude à la coagulation du lait (Mahaut et al., 2000). L'obligation de la pasteurisation n'est pas exigible dans le cas des fromages fermiers au lait cru (Goudédranche et al., 2001). Toutefois, la non-maîtrise du barème de pasteurisation peut rendre difficile l'appréciation de l'efficacité du traitement thermique. Le lactosérum appelé «Magunjo» dans la langue «Shi» est utilisé comme ferment par 100\% des fabricants 


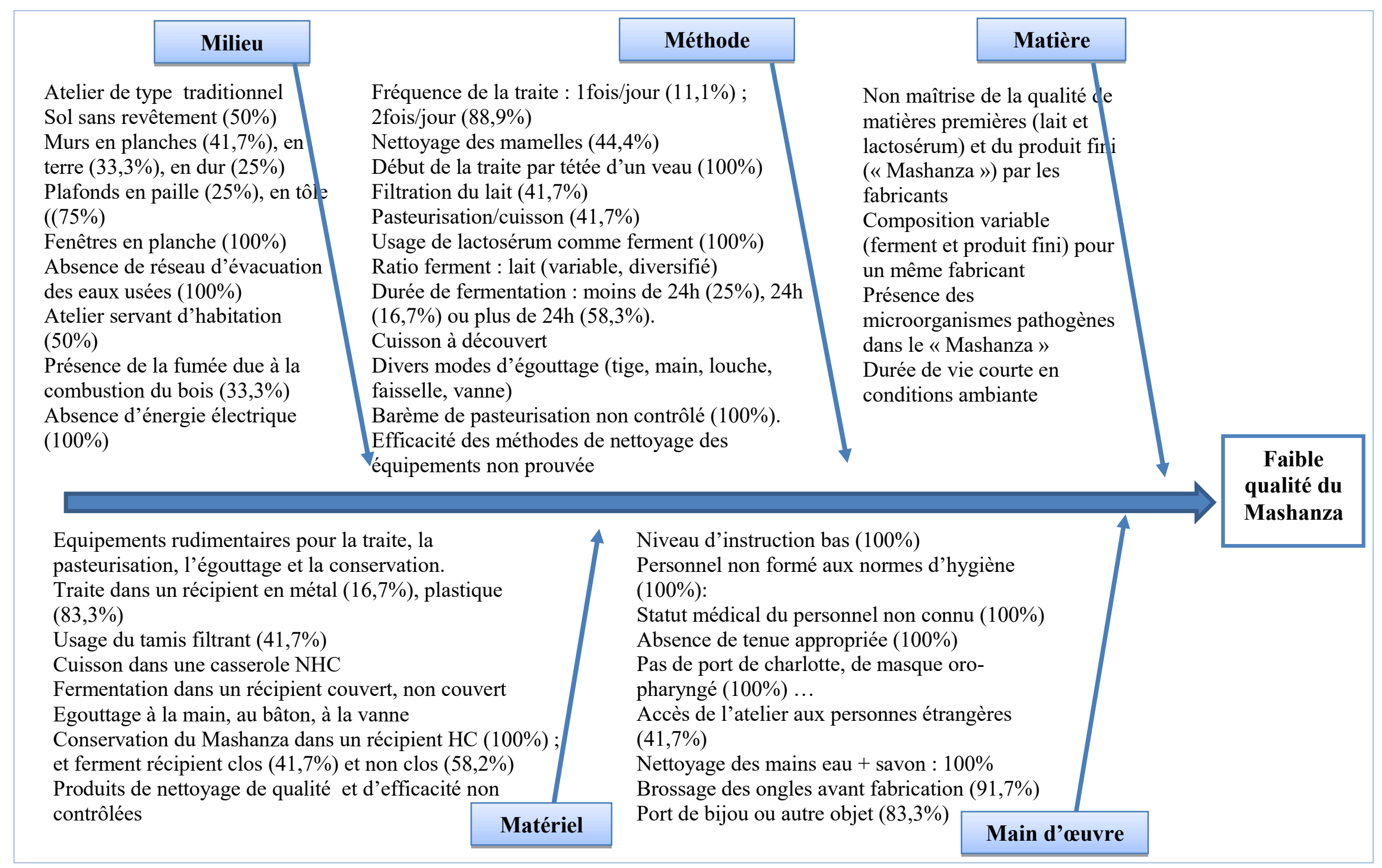

Figure 2 : Facteurs influençant la qualité du fromage frais Mashanza dans 12 unités de fabrication du Sud-Kivu 
En effet, les ferments indigènes tels que le lactosérum contribuent à la typicité des produits en apportant des flores spécifiques et diverses les rendant plus résistants aux attaques phagiques que dans le cas de l'utilisation d'autres ferments (Anonyme, 2016). Pour les critères d'appréciation de la qualité de ferment lactique, $50 \%$ de fabricants apprécient le ferment par l'absence de coagulum dans le ferment, $41,7 \%$ par son goût (piquant) et son odeur et $8,3 \%$ par son aptitude à la coagulation. Selon Anonyme (2016), en technologie lactique, le lactosérum utilisé comme ferment doit provenir d'un caillé de bonne qualité, évaluée par son apparence, son odeur, sa couleur, son goût ou son $\mathrm{pH}$, l'acidité du lactosérum surnageant et sa température de stockage. Concernant la quantité de ferment utilisée pour 10 litres de lait: 25\% utilisent respectivement 4 et 8 litres ; 16,7\% utilisent respectivement 5 et 7 litres, enfin $8,3 \%$ utilisent respectivement 3 et 6 litres. Le ratio ferment : lait est aussi variable d'une ferme à une autre ou d'une localisation à une autre, excepté à Uvira où le ratio $5: 10$ est standard dans les 3 unités en étude. La durée de fermentation va de 1 jour jusqu'à 7 jours; elle dure moins de 24 heures pour 25\% de fabricants, 24 heures pour $16,7 \%$ de fabricants et plus de 24 heures pour 58,3 \% de fabricants. Le temps de coagulation totale varie de 14 à 48 heures pour les pâtes fraîches, selon Mahaut et al. (2000). Enfin, le mode d'égouttage et le conditionnement du produit dépendent de l'équipement utilisé (cuve munis de vanne, tige en bambou, louche, faisselle, à la main) et surtout des quantités de lait disponible.
Toutefois, l'égouttage à l'aide d'une tige est plus fréquente (32, 3\% de fabricants).

4.2.4 Main d'œuvre: Les résultats ont montré que $100 \%$ des fabricants ne mangent et ne fument pas pendant la fabrication. En outre, $58,3 \%$ des fabricants permettent aux personnes extérieures (visiteurs) de circuler dans la salle de fabrication. On remarque que $91,7 \% \%$ des fabricants brossent les ongles et nettoient les mains à l'eau froide et au savon avant la fabrication du «Mashanza », tandis que 8,3\% ne le font pas. En outre, $100 \%$ d'enquêtés ne portent ni filet à cheveux, ni masque bucconasal, ni blouse ou autres vêtements de travail pendant la fabrication. Par ailleurs, 83,3\% des fabricants portent des articles de bijouterie. Il est interdit de boire et de fumer dans les aires de production des denrées alimentaires. Toute circulation humaine peut être source de contamination par les mouvements d'air qu'elle provoque (Quittet et Nelis, 1999). En effet, L'homme est la principale source de microorganismes dans tous les cas où la biocontamination doit être maîtrisée (Combert, cité par Bimbenet et al., 2007). La biocontamination provoquée par le personnel peut avoir 2 origines: rhinopharyngée et cutanée. Selon ces auteurs, on y remédie par le port de masque oro-pharyngé, des gants, des charlottes, d'un vêtement de travail et enfin par la formation aux règles d'hygiène. Enfin, le port d'articles de bijouterie est proscrite car en plus de tomber dans et/ ou sur les aliments, ils sont porteurs de germes pathogènes (Quittet et Nelis, 1999).

4.2.5 Matière: Le tableau 3 donne les moyennes des résultats du fromage 
Tableau 3 : Résultats des paramètres physico-chimiques du Mashanza

\begin{tabular}{|c|c|c|c|c|c|}
\hline \multirow{2}{*}{ Milieu } & \multirow{2}{*}{ UT } & \multicolumn{4}{|c|}{ Fromage Mashanza } \\
\hline & & MS (\%) & $\mathrm{pH}$ & Aw & Acidité $\left({ }^{\circ} \mathrm{D}\right)$ \\
\hline \multirow{3}{*}{ Uvira } & Kond & 35,5 & 3,5 & 0,79 & 65,9 \\
\hline & Shu & 35,5 & 3,6 & 0,93 & 71,1 \\
\hline & Zia & 31,2 & 3,6 & 0,89 & 51,8 \\
\hline \multirow{3}{*}{ Kalehe } & Mag & 38,3 & 3,7 & 0,88 & 59 \\
\hline & Luk & 31,1 & 3,6 & 0,84 & 74,6 \\
\hline & Luz & 42,4 & 3,6 & 0,84 & 61 \\
\hline \multirow{3}{*}{ Kabare } & Miti & 48,8 & 3,9 & 0,81 & 50,5 \\
\hline & Ucb & 36,2 & 3,7 & 0,81 & 67,2 \\
\hline & Comis & 38,3 & 3,5 & 0,89 & 94,4 \\
\hline \multirow{3}{*}{ Walungu } & $\mathrm{Cab}$ & 36,9 & 3,8 & 0,93 & 100,8 \\
\hline & Cing & 34,9 & 3,7 & 0,95 & 68,8 \\
\hline & Uprelo & 47,1 & 3,5 & 0,92 & 41,5 \\
\hline \multicolumn{2}{|c|}{ Moyenne } & 38 & 3,6 & 0,87 & 67,2 \\
\hline \multicolumn{2}{|c|}{ Maximum } & 48,8 & 3,9 & 0,95 & 100,8 \\
\hline \multicolumn{2}{|c|}{ Minimum } & 31,1 & 3,5 & 0,79 & 41,5 \\
\hline
\end{tabular}

Le contenu en matière sèche est dans la gamme de $31,1 \%$ à $48,8 \%$; le $\mathrm{pH}$ variant de 3,5 à 3,9 ; l'Aw de 0,87 à 0,95 et l'acidité de 41,5 à $100,8^{\circ} \mathrm{D}$. Les pâtes fraîches, sont caractérisées par un $\mathrm{pH}$ compris entre 4,3 et 4,5 et une aw entre 0,980 et 0,995 (Ramet, 1985 ; FAO, 1998 ; Mahaut et al., (2000). Le contenu en matière sèche est faible et se situe entre 20 et $25 \%$ (FAO, 1998). Selon Cuvillier (2005), pour le fromage frais, à un pH au moulage de 4,5-4,6 peuvent correspondre les valeurs d'acidité de $50-65^{\circ} \mathrm{D}$ (fromage lactique). Or les valeurs de $\mathrm{pH}$ de cette étude étant plus basses, on peut donc s'attendre aux valeurs d'acidité plus élevées. Quelques fromages africains sont dans la gamme des valeurs préconisées par les auteurs cités. On peut citer Ayib, avec une teneur en eau de 76\%, (O’Connor, 1993) ; Jben, dont les valeurs de $\mathrm{pH}$ sont dans l'intervalle de 4,18 à 4,26 et l'acidité, de 76 à $83^{\circ} \mathrm{D}$ (Rhiat et al., 2013) ; enfin, Quesillo dont le taux d'humidité peut varier de 60-70\% (FAO, 1990). Les études sur le Mashanza réalisées par Mushayuma \& Cirhuza cités par Birali ${ }^{1}$ \& Cie (2019) ont montré des valeurs proches de celles trouvées dans cette étude, mais s'écartant légèrement de celles préconisées par FAO (1990), Mahaut \& Cie (2000) et Ramet (1985). Ainsi, les valeurs de $\mathrm{pH}$ bas $(<4,5)$, de teneur en eau faible $(<75 \%)$, d'Aw faible $(0,998)$ peuvent influencer la qualité hygiénique et la durée de vie du produit. Le tableau 4 présente les résultats de l'analyse microbiologique des lactosérums et Mashanza. Ainsi on remarque que les lactosérums prélevés dans les différentes unités contiennent une flore aérobie mésophile totale variant de 4 à $8.10^{2}$ $\mathrm{ufc} / \mathrm{ml}$. Les coliformes totaux varient de 0 à $3.10^{2} \mathrm{ufc} / \mathrm{ml}$, les coliformes fécaux, de 0 à $1,6.10^{2} \mathrm{ufc} / \mathrm{ml}$; E.coli de 0 à $11,5.10^{3} \mathrm{ufc} / \mathrm{ml}$; S.aureus de 0 à $2,7.10^{2} \mathrm{ufc} / \mathrm{ml}$, les germes anaérobies sulfito-réducteurs, de 0 à $6.10^{2}$ $\mathrm{ufc} / \mathrm{ml}$. 
Tableau 4 : Concentration des microorganismes trouvés dans le lactosérum et le fromage Mashanza après analyses microbiologiques des échantillons des fermes des différents territoires

\begin{tabular}{|c|c|c|c|c|c|c|c|c|c|c|c|c|}
\hline Microorganisme & \multicolumn{3}{|c|}{ Kabare } & \multicolumn{3}{|c|}{ Uvira } & \multicolumn{3}{|c|}{ Kalehe } & \multicolumn{3}{|c|}{ Walungu } \\
\hline Lactosérum & Ucb & Miti & Comis & Zia & Kond & Shuk & Luk & Luz & Mag & Cing & $\mathrm{Cab}$ & Uprelo \\
\hline Flore aérobie mésophile totale & 14 & 5 & 5 & $8.10^{2}$ & $3.10^{2}$ & 76 & 4 & 61 & 20 & 11 & $2,5.10^{2}$ & 58 \\
\hline Coliformes totaux & 0 & 0 & 25 & 9 & 0 & 5 & 3 & 0 & 0 & 0 & $3.10^{2}$ & 0 \\
\hline Coliformes fécaux & 0 & 0 & 0 & 0 & 0 & 0 & 0 & 0 & 0 & 0 & $1,6.10^{2}$ & 0 \\
\hline Escherichia coli & 0 & 0 & 5 & 3 & $11,5.10^{3}$ & 0 & 0 & 0 & 0 & 0 & 0 & $19.10^{2}$ \\
\hline Staphylococcus aureus & 0 & 0 & 0 & 0 & 0 & 0 & 0 & 0 & 0 & 0 & 273 & 0 \\
\hline Anaérobies sulfito- réducteurs & 0 & 67 & 0 & 0 & $6.10^{2}$ & 15 & 26 & 13 & 25 & 61 & 5 & 39 \\
\hline Levures et moisissures & 45 & 18 & $1,3.10^{2}$ & 45 & $2,3.10^{2}$ & $10^{3}$ & $1,7.10^{3}$ & 0 & 45 & $4,6.10^{2}$ & $3,2.10^{2}$ & $3.10^{2}$ \\
\hline Lactobacillus sp & $4.10^{2}$ & 64 & $8,6.10^{2}$ & $9,1.10^{2}$ & $2,1.10^{3}$ & 345 & $2,7.10^{3}$ & 91 & 36 & $1,5.10^{3}$ & $1,3.10^{3}$ & $1,1.10^{3}$ \\
\hline Salmonella sp & 0 & 0 & 0 & 0 & 0 & 0 & 0 & 0 & 0 & 0 & 0 & 0 \\
\hline \multicolumn{13}{|l|}{ Mashanza } \\
\hline Flore aérobie mésophile totale & 0 & 18 & 0 & 9 & 0 & 0 & 0 & 9 & 73 & $9,8.10^{2}$ & $1,2.10^{3}$ & $9.10^{2}$ \\
\hline Coliformes totaux & 0 & $1,4.10^{2}$ & 0 & 0 & 0 & 0 & 0 & 0 & 0 & 0 & 0 & 0 \\
\hline Coliformes fécaux & 36 & 0 & 0 & 0 & 0 & 0 & 0 & 0 & 0 & 0 & 0 & 0 \\
\hline Escherichia coli & 0 & 0 & 0 & 0 & 0 & 0 & 0 & 0 & 9 & 0 & 0 & 164 \\
\hline Staphylococcus aureus & 0 & 0 & 0 & 0 & 0 & 0 & 0 & 0 & 0 & 0 & 0 & 0 \\
\hline Anaérobie sulfito réducteurs & 36 & 0 & 27 & 0 & 0 & 0 & 0 & 0 & $2.10^{2}$ & $10^{2}$ & $1,2.10^{3}$ & $2,3.10^{2}$ \\
\hline Levures \& moisissures & 0 & 36 & 18 & 18 & $10^{2}$ & 7.10 & 18 & 18 & $2,2.10^{2}$ & 82 & $9,6.10^{2}$ & $1,5.10^{3}$ \\
\hline Salmonella sp & 0 & 0 & 0 & 0 & 0 & 0 & 0 & 0 & 0 & 0 & 0 & 0 \\
\hline
\end{tabular}


Les lactobacilles, de $6,4.10$ à $2,7.10^{3}$ et les levures et moisissures, de 0 à $1,7.10^{3} \mathrm{ufc} / \mathrm{ml}$. Les salmonelles sont absentes dans tous les échantillons Dans le «Mashanza», la flore microbienne se trouve dans la gamme de valeurs pour la flore aérobie mésophile totale allant de 0 à $9,8.10^{2} \mathrm{ufc} / \mathrm{g}$; les coliformes totaux, de 0 à $1,4.10^{2} \mathrm{ufc} / \mathrm{g}$, les coliformes fécaux, de 0 à $36 \mathrm{ufc} / \mathrm{g}$, E. coli, 0 à $1,6.10^{2} \mathrm{ufc} / \mathrm{g}$; les anaérobies sulfito-réducteurs, de 0 à 1,2.103, ufc/g; les levures et moisissures, de 0 à 1,5.103. ufc/g. Quant aux salmonelles et à $S$. aureus, ils sont absents de tous les échantillons. On observe, cependant une réduction de la charge de microorganismes dans le «Mashanza », quoique dans certains cas pour quelques fermes, on peut noter l'apparition des microorganismes à risque, sans doute à cause des défauts d'hygiène et des pratiques non conformes. Par exemple, la qualité de la matière première utilisée, l'absence de traitement thermique ou le choix d'un barème inadéquat, la durée de la fermentation, l'usage des récipients non couverts, ou incorrectement assainis, un environnement ou un personnel contaminé, l'égouttage à la main,autant des facteurs qui peuvent favoriser la présence des germes pathogènes ou d'altération dans la matière première ou le produit fini. La norme pour la flore aérobie mésophile totale doit être $<106 \mathrm{ufc} / \mathrm{g}$ selon les normes françaises publiées par le règlement 2073/2005/CE. De ce fait, les Mashanza produits dans ces territoires se situent dans les normes de la flore aérobie. Pour le fromage frais au lait cru ou au lait thermisé, il est recommandé, pour les salmonelles, une absence dans $1 \mathrm{~g}$, pour $S$. aureus, un seuil maximal de $10^{3} / \mathrm{g}$ et pour E.coli, un seuil de $10^{4} / \mathrm{g}$ d'échantillon (Goudédranche et al, 2001, AFSSA, 2008). Un nombre élevé des coliformes est prévisible dans la mesure où le lait après la traite est le plus souvent souillé par des germes d'origines fécales (Vignola, 2002). La présence des Staphylococcus aureus peut aussi se justifier par la présence d'une mamelle malade chez l'animal qui produit le lait (Ngassam, 2007). La présence quantitative des bactéries lactiques dans les produits fermentés où elles dépassent généralement $10^{6}$ bactéries/g d'aliment exerçant un phénomène de compétition vis-à-vis des autres flores et des propriétés spécifiques d'inhibition s'exerçant alors (Pilet et al, 2005 ; Jamet, 2009 ; Par contre, la production d'acide lactique favorise le développement des microorganismes acidophiles, particulièrement les levures et moisissures (Jamet, 2005). De manière générale, les toxi-infections alimentaires à Clostridium perfringes, $C$. botulinum, E.coli, $S$. aureus et salmonelles sont dues au non respect des règles d'hygiène, associé à des pratiques non conformes (par exemple le respect de la chaîne du froid pour $S$. aureus et salmonelle, lavage des mains,..) (Fach et Perelle, 2005; VernozyRozand, 2005; De Buyser et Sutra, 2005; Humbert, 2005), quoique pour S.aureus, la salubrité des aliments dépend plus du critère entérotoxine que du critère staphylocoque (Humbert, 2005).

4.3 Qualité et perspectives technologiques : La qualité du Mashanza est tributaire de plusieurs facteurs comme illustré à la figure 1. Etant donné que les infrastructures ne sont pas conformes aux normes, des adaptations devraient être envisagées localement compte tenu des matériaux disponibles et des moyens financiers souvent limités en vue d'améliorer les ateliers servant de fabrication du Mashanza et permettre ainsi de garantir la salubrité de l'environnement et la qualité sanitaire du produit. Les efforts similaires devraient être réalisés pour les équipements, l'ensemble des matières premières et $\mathrm{du}$ produit fini ainsi que le personnel travaillant. Les installations sanitaires devraient être prévues et des points d'eau à proximité afin de rendre possible les pratiques d'hygiène. De plus, le management dans ces unités semble se focaliser uniquement sur la production et minimiser l'investissement dans le renforcement des capacités de la main d'œuvre. La faible production ne motive certainement pas d'efforts supplémentaires dans l'investissement, comme un cercle vicieux. Aussi, en améliorant la qualité globale du Mashanza ou en l'adaptant au goût d'autres 
consommateurs moins ancrés dans la tradition du peuple shi, peut-on espérer augmenter sa consommation sous des formes variées et inciter les producteurs à investir dans une production à grande échelle et plus modernisée des fromages dérivés du «Mashanza »? Si la diversification permet d'atteindre une proportion plus large des consommateurs, elle peut certainement inciter plus d'intérêt de la part des fermiers à développer cette filière pour générer plus de revenus, cela d'autant plus qu'il existe plusieurs procédés pour ce produit comme démontré par Birali \& Cie (2019), sans que cela ne génère de nouvelles opportunités. Etant donné la nécessité d'améliorer la qualité sanitaire, sécuritaire et organoleptique du Mashanza et de contrôler les conditions de production, les unités de fabrication devraient adhérer au processus d'application de la réglementation et d'initiation aux bonnes pratiques d'hygiène et aux bonnes pratiques de fabrication. Ce qui suppose, de la part des gestionnaires, une démarche volontaire vers l'acquisition d'une formation sur l'hygiène alimentaire ainsi que sur un ensemble d'outils de gestion de la qualité de manière générale et la maitrise des normes locales comme base de référence si les normes internationales s'avèreraient trop exigeantes au début du processus. Cette démarche serait un préalable pour la mise en place du plan HACCP cadrant avec la réalité de chaque milieu. Selon Torkar et Teger cités par Ndungu \& Cie (2016),

\section{CONCLUSION}

Cette étude visait à diagnostiquer les pratiques d'hygiène et de fabrication du Mashanza au sein de 12 unités de fabrication à Kabare, Kalehe, Uvira et Walungu et a permis d'analyser sommairement la qualité du Mashanza produit dans le cadre de la normalisation du produit. Les résultats ont montré que la majorité des unités de fabrication sont de type artisanal et ne sont pas conformes par rapport aux normes, en général en ce qui concerne les installations, l'aménagement, l'assainissement, les pratiques d'hygiène et de fabrication... Les équipements utilisés sont rudimentaires. Le personnel est peu pour assurer la sécurité alimentaire et réduire les risques, il faudrait mettre en place le système HACCP (Hazard Analysis Critical Control point) ou analyse des dangers et maîtrise des points critiques ; et celui d'assurance qualité à la ferme. Cependant la mise en place du système HACCP, selon le Codex Alimentarius, exige au début la mise en œuvre de bonnes pratiques (Sikora et Nowicki, 2007). Muffok \& Cie (2013) ont montré que L'ISO 22000 est la seule norme internationale qui harmonise les pratiques de management de la sécurité des denrées alimentaires suivant des exigences essentielles comme les PRP et le HACCP afin de garantir l'innocuité des aliments. Toutefois, Louis Bonne (2013), a montré le difficultés de mise en place d'un Plan de Maîtrise Sanitaire (PMS) basé sur des bonnes pratiques d'hygiène et de fabrication (BPH/BPF) et la méthode HACCP du fait de la complexité des documents et méthodes d'application. Dans le cas de cette étude, la recherche de la qualité peut nécessiter que les unités qui sont étrangères aux démarches précitées, s'adaptent aux nouvelles exigences de la qualité, surtout en ce qui concerne l'hygiène alimentaire. Au fur et à mesure qu'elles se développent et visent à accroitre leur échelle de production, qu'elles daignent s'investir davantage, en moyens matériel, financier et humains, ...en vue d'atteindre les niveaux d'exigence de plus en plus élevés.

instruit et routinier, et n'a pas été formé aux normes d'hygiène ni aux diverses procédures. Des efforts devraient être fournis afin de développer cette spécialité et produire à grande échelle un fromage de qualité garantie pouvant répondre aux exigences du marché tant local qu'international. Une initiation à l'application des bonnes pratiques d'hygiène et des bonnes pratiques de fabrication est une démarche préalable qui permettrait aux unités de fabrication, soucieuse d'améliorer la qualité du "Mashanza », d'offrir aux consommateurs des produits sains tout en faisant face à la 
concurrence. Par conséquent, cette démarche suppose le concours d'une main d'œuvre formée et capable de répondre à certaines exigences face à la recherche continuelle de la qualité.

\section{RÉFÉRENCES BIBLIOGRAPHIQUES}

Agence Française de Sécurité Sanitaire des Aliments (AFSSA): 2008. Avis de l'Agence française de sécurité sanitaire des aliments concernant les références applicables aux denrées alimentaires en tant que critères indicateurs d'hygiène des procédés. www.anses.fr/fr/system/files/MIC200 7sa0174.pdf. $21 \mathrm{pp}$

Anonyme: 1993. Mémento de l'Agronome. Ministère de la coopération, $4^{\text {ème }}$ Edition, France. 1635pp.

Anonyme: 2009. Spécification technique de l'achat public : Lait et produits laitiers. Groupes d'étude des marchés de restauration collective et de nutrition (GEMRCN). www.economie.gouv.fr/daj/specificatio n-technique-ndeg-b3-07-09-applicableaux-laits-et-aux-produits-laitiers-date. $47 \mathrm{pp}$.

Anonyme: 2016. Guide européen de bonnes pratiques d'hygiène en production des fromages et produits laitiers artisanaux. Secteur concerné : producteurs fermiers et artisans : France. www.ec.europa.eu/food/sites/food/fil es/safety/docs/biosafety fh guidance artisanal-cheese-and-dairyproducts fr.pdf. 103pp.

Bimbenet JJ, Duquenoy A et Trystram G, 2007 : Génie des procédés alimentaires. Des bases aux applications. Dunod, Paris. 567pp.

Birali M, Sumbu ZE et Walangululu MJ: 2019. « Note de recherche : Fabrication du fromage frais «Mashanza» produit à Bwegera (Uvira), Kabare, Kalehe et Walungu ». Revue des sciences agronomiques et d'ingénierie biologique appliquée du Kivu, Harmattan, p.

Birali M, Sumbu ZE et Walangululu MJ: 2019: 2019. Diagnostic des procédés de fabrication $\mathrm{du}$ fromage blanc traditionnel «mashanza» dans les zones productrices du Sud-Kivu, J. Appl. Biosci. 138: 14017 - 14028.

Cuvillier D: 2005. Améliorer le rendement fromager.

www.cfbourgogne.free.fr/IMG/pdf/fic he_ameliorer_rendement.pdf. 6pp.

De Buyser ML et Sutra L: 2005. Staphylococcus aureus. in : BIBLIOGRAPHY Bactériologie alimentaire. Compendium d'bygiène des aliments, 292pp. Economica, Paris. 26-51pp.

FAO: 1990. The technology of traditional milk products in developing countries. www.fao.org/docrep/003/t0251e/T02 51E00.htm\#TOC. 223pp.

FAO: 1998. Le lait et les produits laitiers dans la nutrition humaine. www.fao.org/docrep/t4280f/T4280F0f .htm\#Chapitre \%206\%20Fromages. $255 \mathrm{pp}$.

FAO: 2011. Principes généraux d'hygiène alimentaire.

www.fao.org/input/download/standar ds/23/CXP_001f.pdf. 29pp.

Fach P et Perelle S: 2005. Clostridium perfringens et C. botulinum. in BIBLIOGRAPHY : Bactériologie alimentaire. 292pp. Compendium d'hygiène des aliments. Economica, Paris. 77-96pp.

Goudédranche H., Camier-Caudron B., Gassi JY et Schuck P: 2001. Procédé de transformation fromagère (partie 1). Technique de l'ingénieur, traité Agroalimentaire. F6305. 11pp.

BIBLIOGRAPHY Humbert F: 2005. Les Salmonelles. Dans: Bactériologie alimentaire. Compendium d'bygiène des aliments. Economica, Paris. 292pp.

BIBLIOGRAPHY \l1036 Jamet E: 2009. Les bactéries lactiques: une composante de l'écosystème microbien des fromages, 
593pp. In: Drider DJ et Prévost H., (Eds.) Bactéries lactiques, Economica, Paris. 319-343pp.

Konte M: 1999. Le lait et les produits laitiers : Développement des systèmes de production intensive en Afrique de l'Ouest. Institut Sénégalais de Recherche Agricole, Sénégal. 25pp.

Louis Bonne R: 2013. Présentation de deux méthodes originales visant à faciliter dans les IAA, la mise en œuvre des bonnes pratiques d'hygiène et de fabrication ainsi que de la méthode HACCP, telles que définies par le Codex alimentarius. "Thèse de Doctorat", Université de Toulouse. 132pp

Mahaut M, Jeantet $R$ et Brulé G: 2000. Initiation à la technologie fromagère. Technique et Documentation, Paris. $194 \mathrm{pp}$.

Ministère du Plan: 2005. Monographie de la province du Sud- Kivu, PNUDUNOPS. 179 pp.

Mouffok N, Benhadja L, Ferhat Z et Bousbia : 2013. Identification et analyse des dangers d'un processus de fromage fondu selon l'ISO 22 000. Qualita, Compiègne. www.hal.archivesouvertes.fr/hal-00823126. 9pp

Ndungu TW, Omwamba M, Muliro PS and Oosterwijk G: 2016. Hygienic practices and critical control points along the collection chains in smallholder collection and bulking enterprises in Nakuru and Nyandarua Countries, Kenya. Afr. J. Food Sci., 10 (11): 327339.

Ngassam T: 2007. Caractérisation de la flore lactique des laits fermentés artisanaux au Sénégal: cas de la zone de Niayes. "Thèse de Doctorat", université Cheikh Anta Diop de Dakar. 109 pp.

O'Connor CB: 1993. Traditional cheesemaking manual, ILCA (International Livestock Centre for Africa), Addis Ababa. 50 pp.

BIBLIOGRAPHY Pilet MF, Magras C et Federighi M: 2005. Bactéris lactiques.
In: Bactériologie alimentaire. Compendium d'bygiène des aliments. Economica, Paris. 219-240pp.

Poret S: 2011. Normes de Qualité dans l'Agroalimentaire. INRA-ALISS et Ecole Polytechnique. Chaire FDIRGT2. Les labels ISR. 29 Juin 2011. Alimentation, agriculture et environnement, France. 10pp.

Quittet C et Nelis H: 1999. HACCP pour PME et artisans. Secteur produits laitiers. Les Presses agronomiques de Gembloux, Belgique. 494 pp.

Ramet JP: 1985. La fromagerie et les variétés de fromages du bassin Méditerranéen. Etude FAO. Production et santé animale www.fao.org/docrep/004/X6551F/X6 551F00.HTM. 187pp.

Rhiat M, Labioui H, Driouich A, Mennane, Z et Ouhssine M: 2013. Preparation of the starter Trial production of cheese (Jben) and Klila at laboratory scale. Food Science and Quality Management. 13: 1-8.

Roudaut H et Lefrancq E: 2005. Alimentation théorique. Sciences des aliments, Doin. 303 pp.

Sikora T and Nowicki P: 2007. Food safety assurance according to Codex alimentarius and ISO 22000 Standard. Pol. J. Food Nutr. Sci., Vol. 57, No. 4(C): 489-493.

Sylvander B: 1996. Normalisation et concurrence internationale : $\mathrm{La}$ politique de qualité alimentaire en Europe. In: Économie rurale. $\mathrm{N}^{\circ} 231$, Les multinationales de l'agroalimentaire.

www.persee.fr/doc/ecoru_00130559_1996_num_231_1_4772. 56-61p.

Vernozy-Rozand C: 2005. Escherichia coli producteurs de shigatoxines (STEC), 292pp, in : BIBLIOGRAPHY Compendium d'hygiène des aliments. Economica, Paris. 53-76pp. 